\title{
Wideband RCS Reduction and Gain Enhancement for a Patch Antenna with Broadband AMC Structure
}

\author{
Yue GE, Yongjiu ZHAO, Jiaqing CHEN \\ College of Electronic Information Engineering, Nanjing University of Aeronautics and Astronautics, \\ Nanjing, Jiangsu, 210006, China \\ yuege_nuaa@foxmail.com
}

Submitted August 8, 2018 / Accepted November 6, 2018

\begin{abstract}
In this paper, a simultaneous improvement of radiating and scattering performance for a patch antenna is realized. For achieving the wideband low-scattering property, a broadband AMC structure with orthogonally slot-embedding is proposed and analyzed, which is arranged around the antenna with another square ring unit in a chessboard configuration for phase cancellation. Meanwhile, a gain increment is also achieved due to the coupling between the radiation patch and the broadband $A M C$ structure. Measured results indicate that at least a $10 \mathrm{~dB}$ RCS reduction is obtained from $11 \mathrm{GHz}$ to $17.8 \mathrm{GHz}$. Additionally, the gain of the antenna has also been increased for more than $2 \mathrm{~dB}$ between $12 \mathrm{GHz}$ and $13 \mathrm{GHz}$.
\end{abstract}

\section{Keywords}

Artificial Magnetic Conductor (AMC), gain, Radar Cross Section Reduction (RCSR)

\section{Introduction}

With the rapid development of detection and stealth technology, Radar Cross Section (RCS) plays an important role in evaluating the stealth performance of a target. For modern stealth platforms, antennas contribute the total RCS significantly. Meanwhile, they undertake the function of radiating and receiving electromagnetic waves. Thus, the realization of RCS reduction for antenna while maintaining the excellent performance and a stable operation is extremely important.

Artificial Magnetic Conductor (AMC) is a kind of Artificial Electromagnetic Material (AEMM) [1-5] with characteristics of a perfect magnetic conductor (PMC), which is periodically arranged by a specific shape of unit cell [6]. Due to the unique property of reflection phase, AMC has been widely applied to antennas in improving the overall performances, such as gain enhancement [7-11] or RCSR [12-19]. In reference [12], a novel frequency selective surface (FSS) is proposed in MIMO antennas design. Both isolation and RCSR have been achieved based on this struc- ture and method. A low observable antenna is proposed in [13] by using active hybrid-element FSS structure. Although the antenna realized both in-band and out-of-band RCSR, it became non-radiating in original radiation band due to the transmitting characteristic of FSS structure. In [14] and [15], the RCS of a patch antenna was reduced by loading a polarization conversion meta-surface. In [16], a quasi-fractal AMC structure combined with perfect electric conductor (PEC) is applied to a slot-coupled patch antenna to achieve an ultra-wideband RCSR. However, the radiation properties of antennas are not improved among the aforementioned works. The improvements of scattering and radiation performances are both obtained in [17] by exploiting a square-shaped AMC with octagonal ring arrangement. But the operation band of the above antenna is relatively narrow. In [18], a chessboard-like metasurface consisting of two different AMC unit cells is combined with a patch antenna. By rotating the Jerusalem AMC units around the antenna properly, both the broadband RCSR and gain enhancement are obtained. A complementary single resonant ring (CSRR) is used in [19] to achieve the in-band RCSR of the patch antenna while effectively increasing the antenna gain, however, the RCSR bandwidth is still narrow.

In this paper, we proposed a broadband AMC structure with orthogonal slot slot-embedding for the simultaneous improvement of radiating and scattering performance of a patch antenna. By adding slots in the conventional dual-loop AMC design, the relative in-phase reflection bandwidth is extended to $50 \%(9 \mathrm{GHz} 15 \mathrm{GHz})$. At the same time, by arranging the broadband AMC and square ring unit surrounding the patch antenna in a chessboard configuration $(\mathrm{CBC})$ for phase cancellation of reflected wave, both remarkable RCSR and gain enhancement are realized over the operating band of the antenna. The design is verified through numerical analysis and experiment, and a good agreement is observed between simulation and measurement. The detailed design of broadband AMC unit is introduced in Sec. 2. Section 3 shows the simulated results for a wideband low scattering patch antenna with and without loading AMC. To validate the design, the patch antenna with AMC is fabricated and measured in Sec. 4. Finally, a summary is given in Sec. 5. 


\section{Broadband AMC Unit Design}

The principle of RCS reduction for CBC lies in the backscattering cancellation which depends on the phase difference of AMC cells, which can be approximated by array theory. According to [19], [20], the RCS of a target is defined as

$$
\sigma=\lim _{r \rightarrow \infty} 4 \pi r^{2} \frac{\left|E_{\mathrm{s}}\right|^{2}}{\left|E_{\mathrm{i}}\right|^{2}}
$$

Assuming the condition in Fig. 1, when a plane wave normally impinges the metasurface, the total reflected energy is a summation of the reflection from all of the blocks.

For an antenna array with both the AMC blocks possessing same elements, the total reflection can be represented by

$$
\begin{gathered}
E_{\text {total }}=E_{\mathrm{AMC} 1} \cdot A F_{\mathrm{AMC} 1}+E_{\mathrm{AMC} 2} \cdot A F_{\mathrm{AMC} 2}, \\
A F_{\mathrm{AMC} 1}=\mathrm{e}^{\mathrm{j}(k x+k y) d / 2}+\mathrm{e}^{\mathrm{j}(-k x-k y) d / 2}, \\
A F_{\mathrm{AMC} 2}=\mathrm{e}^{\mathrm{j}(k x-k y) d / 2}+\mathrm{e}^{\mathrm{j}(-k x+k y) d / 2}
\end{gathered}
$$

where $A F_{\mathrm{AMC} 1}$ and $A F_{\mathrm{AMC} 2}$ are the array factors of the different AMC structures, respectively. $k$ is the wave number and $d$ is the central distance between adjacent elements, which can be denoted as

$$
\begin{gathered}
k=\frac{2 \pi}{\lambda}, \\
k x=k \sin \theta \cos \varphi, \\
k y=k \sin \theta \sin \varphi .
\end{gathered}
$$

Compared to a metal plate, the RCS reduction of a scattering metasurface can be expressed by

$$
\text { RCS reduction }=10 \log \left[\frac{\lim _{r \rightarrow \infty}\left[4 \pi r^{2}\left|\frac{E_{\mathrm{s}}}{E_{\mathrm{i}}}\right|^{2}\right]}{\lim _{r \rightarrow \infty}\left[4 \pi r^{2}(1)^{2}\right]}\right] .
$$

For a dual AMC, chessboard surface can be approximated by

$$
R C S R=10 \log \left[\frac{A_{1} \mathrm{e}^{\mathrm{j} \phi_{1}}+A_{2} \mathrm{e}^{\mathrm{j} \phi_{2}}}{2}\right]^{2}
$$

where $A_{1}$ and $A_{2}$ are the reflection coefficient amplitudes of two AMC structures, and $\Phi_{1}$ and $\Phi_{2}$ are their reflection phase. According to the above equations, a $10 \mathrm{~dB}$ RCS reduction can be achieved when the phase difference satisfies the condition with the same amplitudes of two AMC cells

$$
\left|\Phi_{\text {AMC1-AMC2 }}\right| \leq 180^{\circ} \pm 30^{\circ}
$$
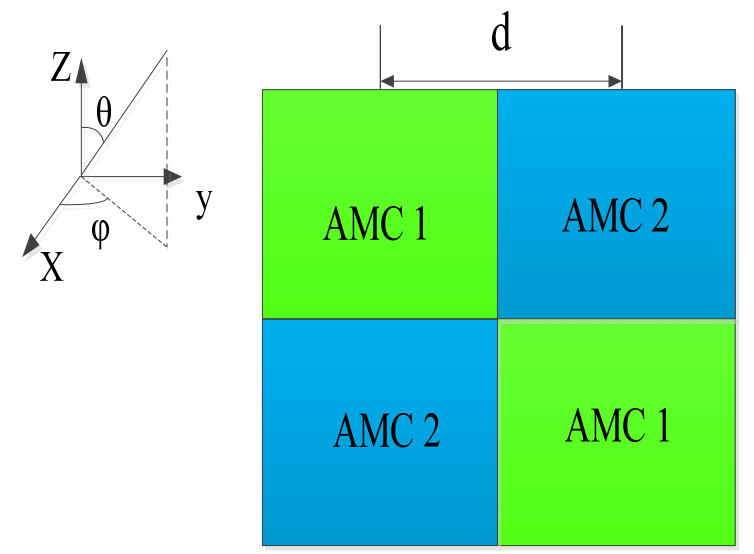

Fig. 1. Geometry of AMC-AMC chessboard.

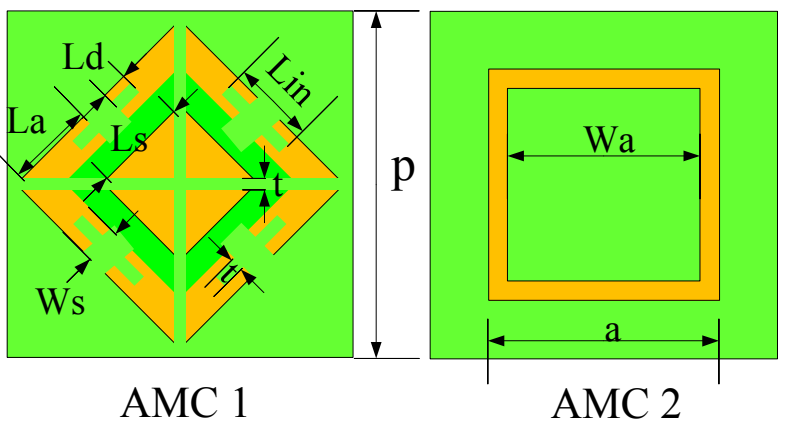

Fig. 2. Geometries of $\mathrm{AMC} 1$ and $\mathrm{AMC} 2$.

It means that a broadband RCSR can be obtained when (10) is satisfied over a wideband frequency range. In order to achieve this goal, two different AMC cells are designed in this paper. The geometry of the proposed AMC unit cells are depicted in Fig. 2, denoted as AMC 1 and AMC 2, respectively. Metallic ground plane on the bottom side is applied to guarantee the absolute reflection. Polytef dielectric slab (FR4) with a thickness of $2.4 \mathrm{~mm}$ is chosen as the substrate. The relative permittivity is 4.4 and the dielectric loss tangent is 0.02 . The same periodicity $p$ is adopted for all AMC cells.

In Fig. 2, AMC 1 is the wideband cell proposed in this paper. In AMC 1, the length of arms $(L a)$, inner patch length $(L s)$ and orthogonal slots $(t)$ have the major influences on the reflection phase bandwidth. The square ring length $(a)$ determines the resonant frequency of AMC 2. Both AMC cells are polarization-independent due to their symmetrical geometries. The proposed broadband AMC 1 is designed based on the parasitic elements technique in wideband structures such as a microstrip antenna with a parasitic patch. The bandwidth enhancement of the AMC is achieved by combining the capacitive and inductive couplings with an equivalent parallel LC resonant circuit, which contributes the effects of arms and parasitic patches. When a plane wave with the electric field along the $\mathrm{x}$-axis direction incidents perpendicularly on the AMC unit, the induced current in the outer resonance arms of the cell can be modeled as an inductance $L$ and the metal part separated by the gap represents the gap capacitance $C$, thus, the AMC unit cell can be characterized by a resonant frequency $f_{0}$ and the in-phase reflection $B W$ as the followings, 


$$
\begin{gathered}
f_{0}=\frac{1}{2 \pi \sqrt{L C}}, \\
B W \propto \sqrt{\frac{L}{C}} .
\end{gathered}
$$

As can be seen from the above equations, the relative bandwidth of the cell is proportional to $L$ and inversely proportional to $C$, which means the bandwidth of AMC cell can be improved either by increasing inductance or decreasing capacitance. To further improve the bandwidth of AMC 1, two orthogonal slots are added to the structure, and the equivalent capacitance of AMC is reduced. Figure 3 shows the equivalent circuit when a plane wave incidents perpendicularly to AMC 1 . And Figure 4 shows the plotted surface current at $12 \mathrm{GHz}$.

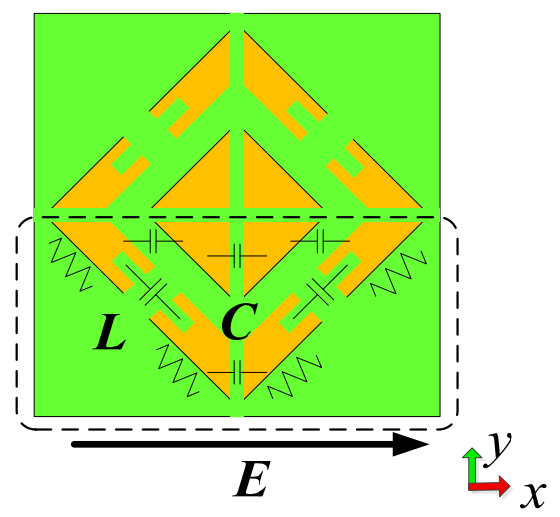

Fig. 3. Equivalent circuit of AMC 1.

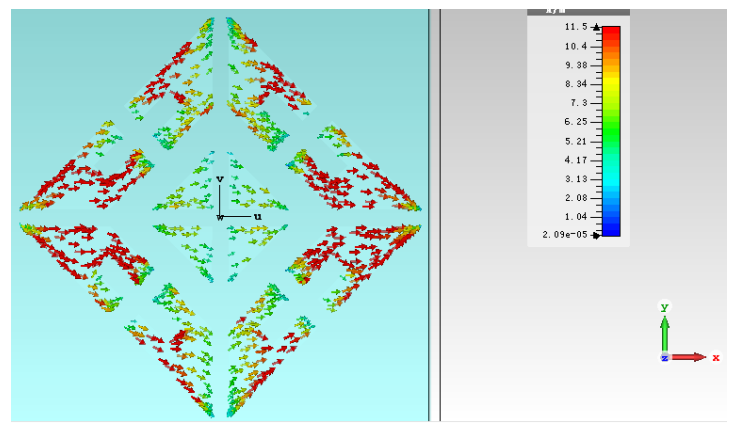

Fig. 4. Surface current of AMC 1 at $12 \mathrm{GHz}$.

According to the plotted surface current of unit in Fig. 4, when the E-field of plane wave is along the $\mathrm{X}$ direction, we can clearly see that the induced surface current was mostly concentrated on its outer arm, which means the arm $L s$ can be replaced by inductance $(L)$ and the gap between parallel parts can be replaced by capacitance $(C)$. To verify the impact of the reflection phase properties for different slot widths, full-wave numerical analysis has been carried out in CST Microwave Studio as shown in Fig. 5. The reflection properties of AMC units can be obtained by defining the Floquet port and unit cell boundary conditions.

The reflection phase of AMC 1 versus frequency for different parameters is illustrated in Fig. 6(a), (b) and (c). It can be found from Fig. 6 that whether the slot width $t$ increases or arm length $L a$ decreases, the resonant frequency

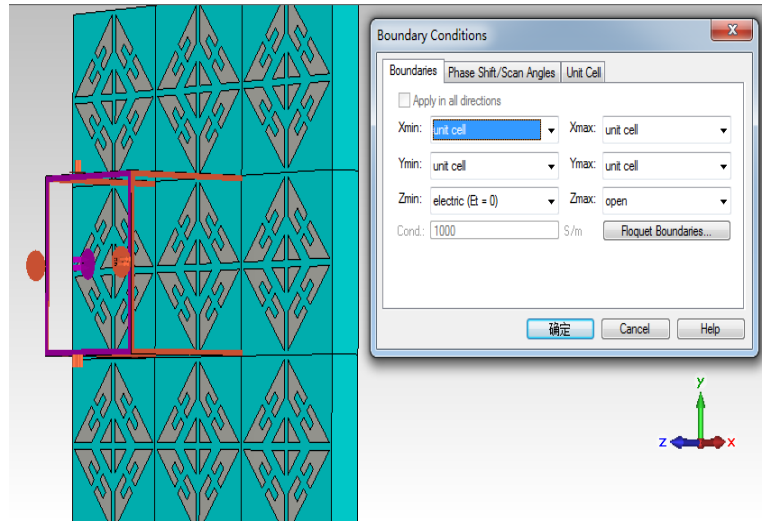

Fig. 5. Model design of AMC in CST.

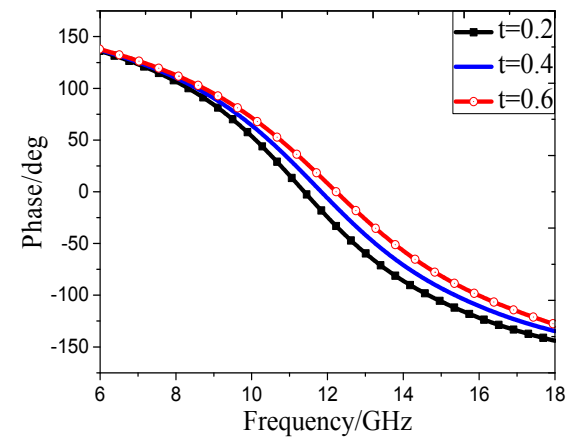

(a)

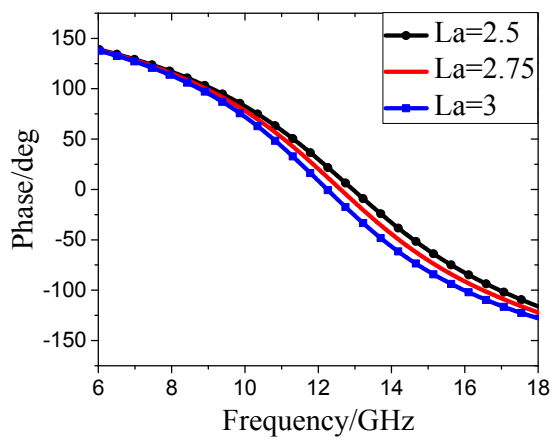

(b)

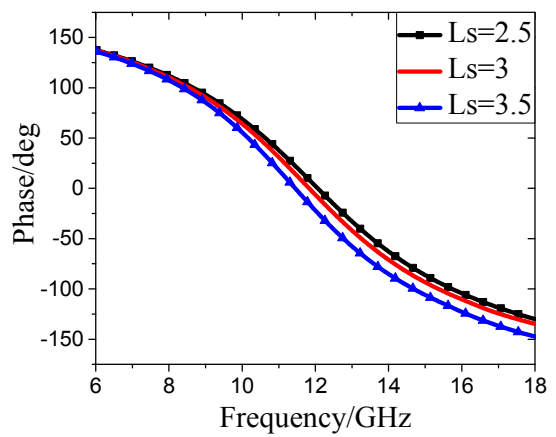

(c)

Fig. 6. Reflection phase versus frequency for different parameters (a) $t$, (b) $L a$, (c) $L s$.

of the unit moves upward and the in-phase reflection bandwidth increases at the same time. Besides, the inner patch length also affects the bandwidth of AMC 1. By adjusting the above parameters properly, the equivalent capacitance and inductance can be changed, then the reflection phase bandwidth of two AMC units can be controlled flexibly. 


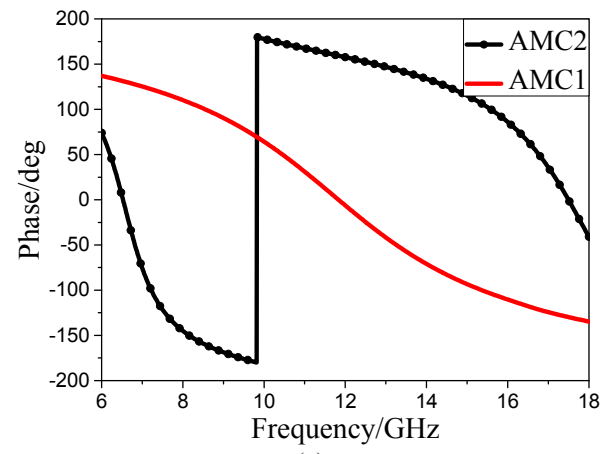

(a)

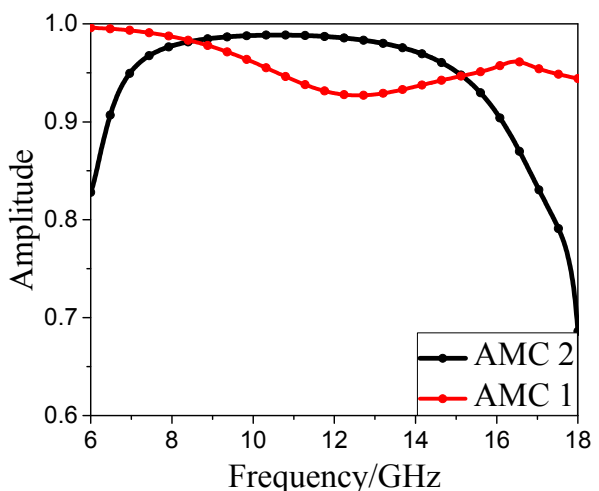

(b)

Fig. 7. Reflection properties of AMC 1 and AMC 2: (a) phase, (b) amplitude.

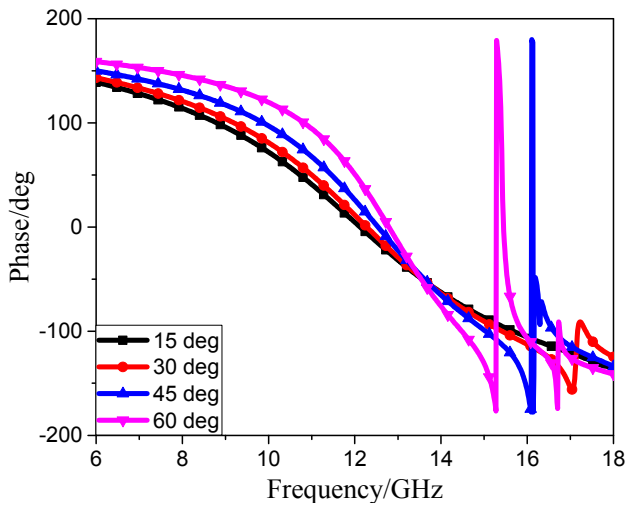

(a)

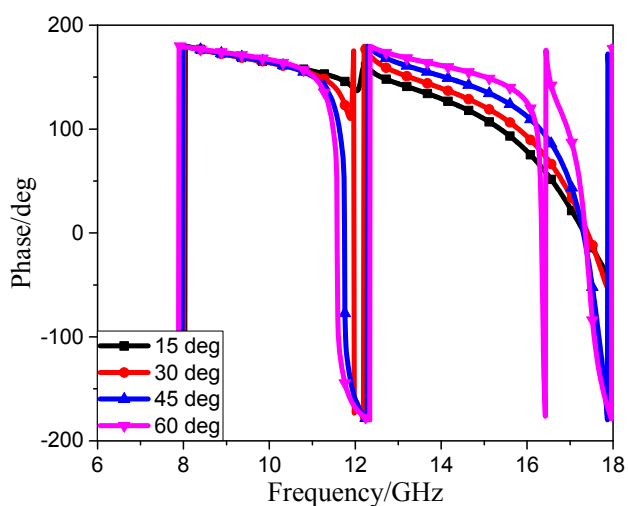

(b)

Fig. 8. Reflection phase of AMC 1 and AMC 2 at different incident angles: (a) AMC 1, (b) AMC 2.
Figure 7(a) and (b) shows the reflection property curves of two units versus frequency, respectively. It can be observed from Fig. 7 that the two AMC cells operate at different frequency bands. The resonance frequency of AMC 1 is $12 \mathrm{GHz}$ and the in-phase reflection bandwidth is $50 \%$. AMC 2 possesses dual resonance frequency at $7 \mathrm{GHz}$ and $17.5 \mathrm{GHz}$, respectively. Besides, the responses of two AMC cells at different incidence angles are also considered in Fig. 8.

From Fig. 8, it can be derived that with the incidence angle coming up, the reflection properties of both AMC cells came to worse. However, the proposed wideband AMC 1 unit possesses better stability at different angles compared to AMC 2, which could be considered applying in wide-angle incidence condition. The simulated reflection phase difference versus frequency curve is plotted in Fig. 9.

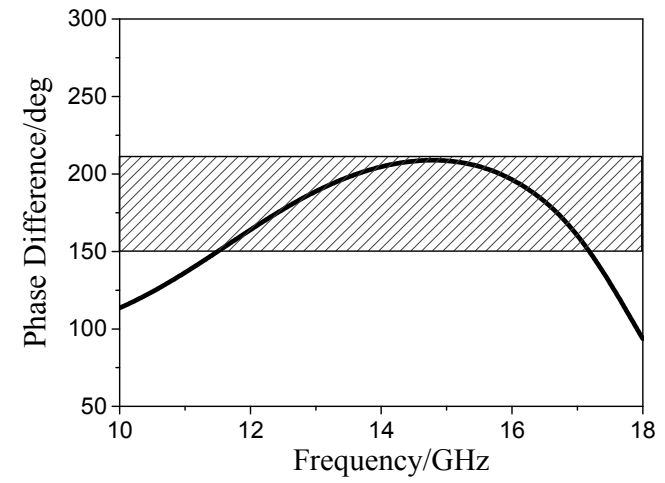

(a)

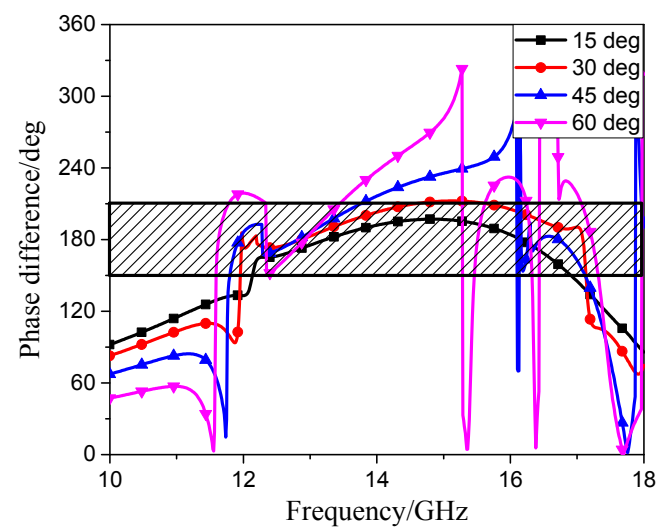

(b)

Fig. 9. Phase difference of AMC 1 and AMC 2: (a) at normal incidence, (b) at oblique incidence angles.

\begin{tabular}{|c|c|}
\hline Dimension & Value $[\mathrm{mm}]$ \\
\hline$W s$ & 1.5 \\
\hline$L a$ & 3.25 \\
\hline$L s$ & 3 \\
\hline$t$ & 0.4 \\
\hline$a$ & 6.8 \\
\hline$W a$ & 0.9 \\
\hline$p$ & 10 \\
\hline$L i n$ & 2.5 \\
\hline$L d$ & 1 \\
\hline
\end{tabular}

Tab. 1. Structure parameters of the AMC 1 and AMC 2. 
According to Fig. 9(a) and (b), a phase difference in the range of $180^{\circ} \pm 30^{\circ}$ can be observed from $11.5 \mathrm{GHz}$ to $17.5 \mathrm{GHz}$, at the same time, the phase difference can still be kept well between $12 \mathrm{GHz}$ and $17 \mathrm{GHz}$ till the angle coming up to $60 \mathrm{deg}$. Finally, the detailed parameters of two AMC units are given in Tab. 1.

\section{Wideband Low Scattering Patch Antenna Design}

A square patch antenna is chosen as a reference antenna. Through comprehensive investigations of the impact of different number of units and loading manners on the radiation performance of antenna, the $\mathrm{CBC}$ with $3 \times 3$ AMC blocks is selected and the schematic view of the proposed antenna is depicted in Fig. 10(a). The substrate applied in antenna design is the same as that used in AMC cells to load AMC units and antenna properly. To validate the design, a prototype of the proposed antenna with AMC is fabricated and shown in Fig. 10(b).

The overall size of the antenna with AMC is $90 \mathrm{~mm} \times 90 \mathrm{~mm} \times 2.4 \mathrm{~mm}$. The comparison between simulated and measured reflection coefficients is presented in Fig. 11.

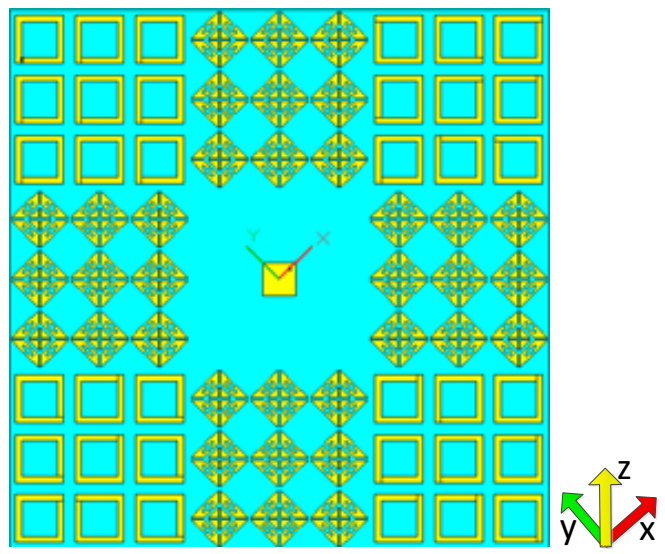

(a)

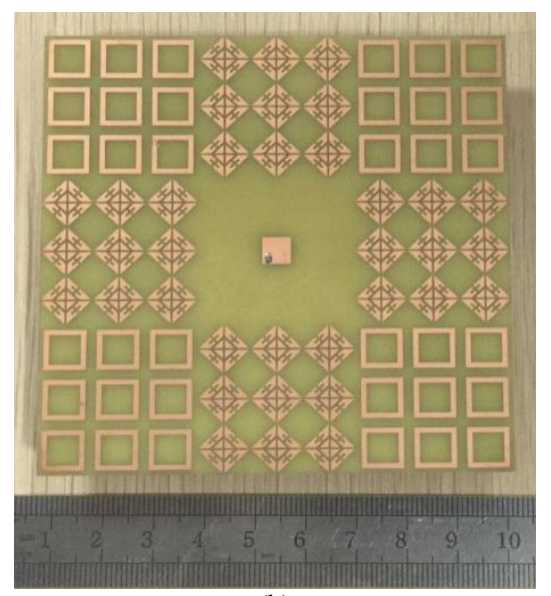

(b)

Fig. 10. Geometry of antenna with AMC: (a) Simulation. (b) Fabrication.

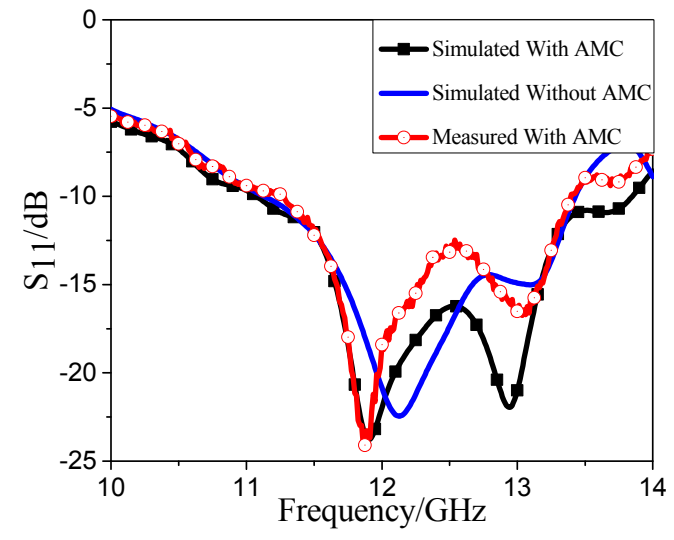

Fig. 11. Simulated and measured reflection coefficients.

In Fig. 11, the measured bandwidth for $S_{11} \leq-10 \mathrm{~dB}$ is around $2 \mathrm{GHz}$, covering from $11.5 \mathrm{GHz}$ to $13.5 \mathrm{GHz}$. A good agreement is observed between simulated and measured results. Figure 12 shows the comparison between the simulated and measured directivity and gain curves versus frequency.

The most important function of AMC in this paper is to realize the RCS reduction, besides, according to the Fig. 12, it can be seen that the directivity and gain of the antenna with AMC are enhanced over the range from $12 \mathrm{GHz}$ to $13 \mathrm{GHz}$, meanwhile, the maximum directivity and gain at $12.4 \mathrm{GHz}$ are $9 \mathrm{dBi}$ and $7.91 \mathrm{dBi}$, respectively.

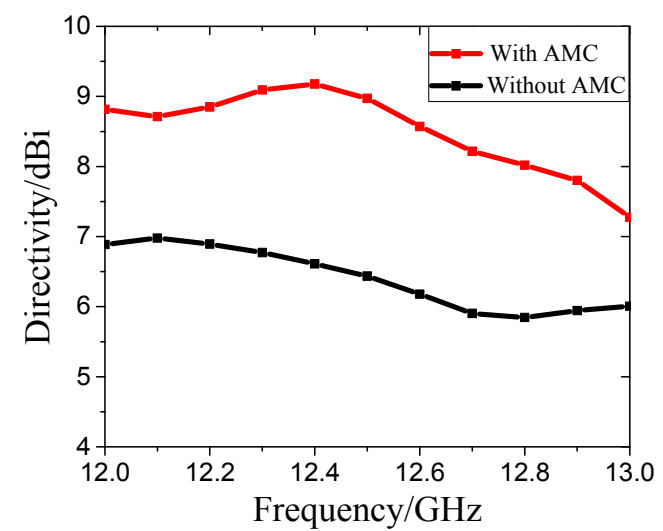

(a)

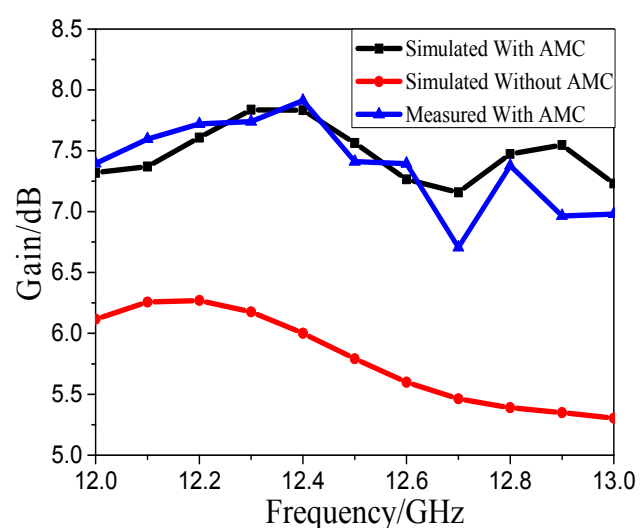

(b)

Fig. 12. Simulated and measured radiation performance: (a) Directivity. (b) Gain. 
Both of them are $2 \mathrm{~dB}$ higher than that of the antenna without AMC over the working range. To further investigate the mechanism of the gain enhancement after loading the $\mathrm{AMC}$, the current distribution of the proposed antenna at 12.4 GHz is shown in Fig. 13 (a), (b).

It can be seen from Fig. 13 (b) that coupling occurs between the proposed AMC 1 and radiation patch at 12.4 GHz. And the coupling is also observed from $12 \mathrm{GHz}$ to $13 \mathrm{GHz}$. Therefore, the radiation aperture is enlarged after loading AMC, which is the main reason of gain enhancement. The measured and simulated E-plane, Hplane and 3D radiation patterns with and without AMC at 12.4 GHz are shown in Fig. 14(a), (b) and (c).

According to Fig. 14, it can be observed that the radiation performance of the antenna with AMC has been improved due to the strong resonance of the broadband AMC. The experimental results agree well with simulation.

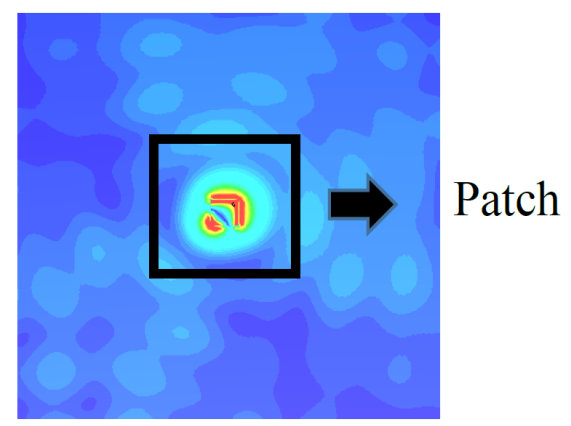

(a)

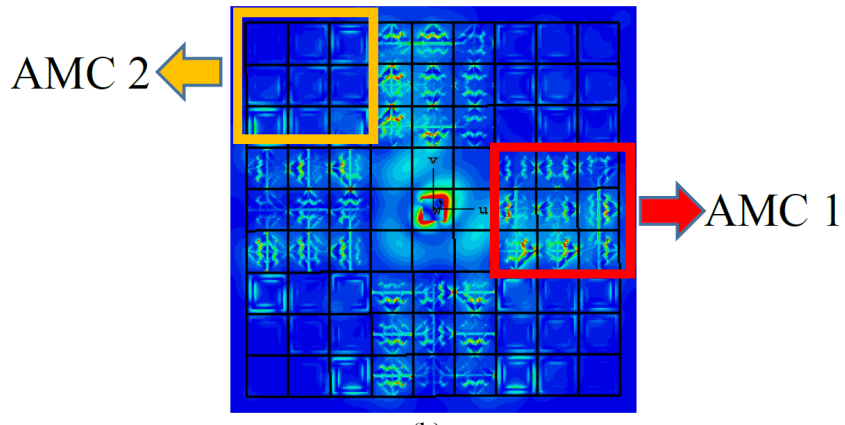

(b)

Fig. 13. Field distribution at $12.4 \mathrm{GHz}$ : (a) without AMC, (b) with AMC.

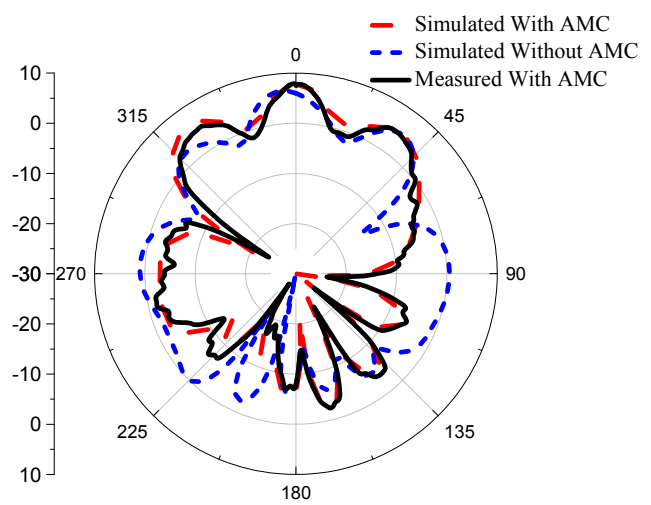

(a)

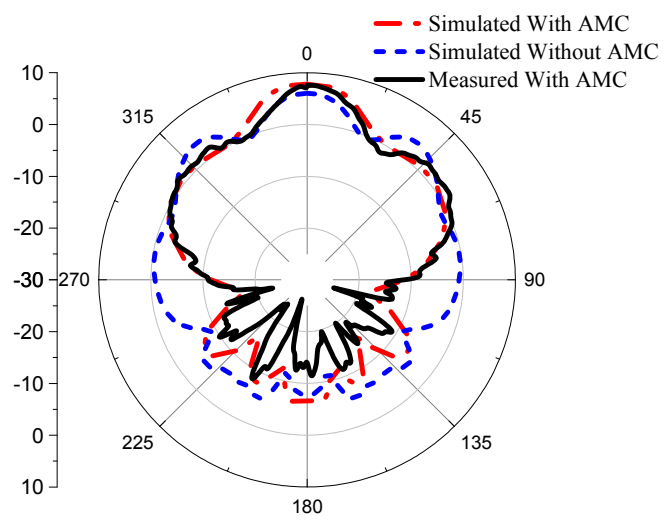

(b)

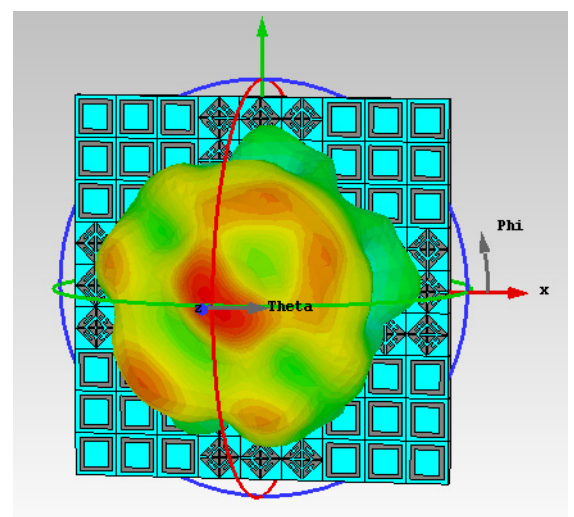

(c)

Fig. 14. Radiation patterns with and without $A M C$ at $12.4 \mathrm{GHz}$ : (a) xoz-plane, (b) yoz-plane, (c) 3D radiation pattern.

\section{Fabrication and Measurement}

For the proposed antenna in this paper, the scattering performances should also be considered as important as the radiation performance. Both the RCS properties of antenna with AMC under TE- and TM- polarization are shown in Fig. 15. According to Fig. 15, the RCS of antenna with AMC under TM-polarization is entirely the same as that under TE-polarization, due to the polarization-independent configuration of both AMC cells. To further confirm the effectiveness of this design, the sample antenna is experimentally characterized.

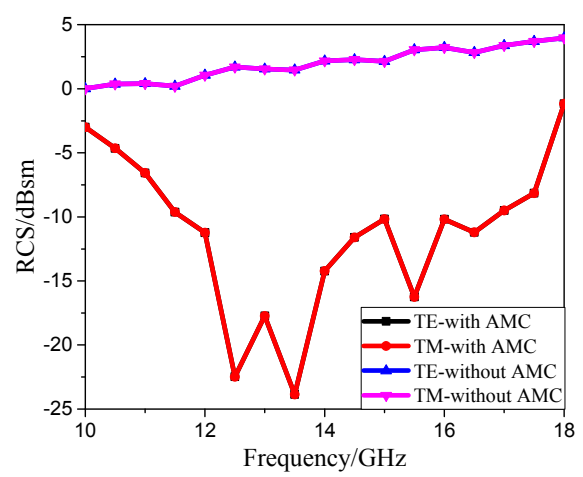

Fig. 15. RCS of the antenna with and without AMC for TE- and TM-polarization condition. 


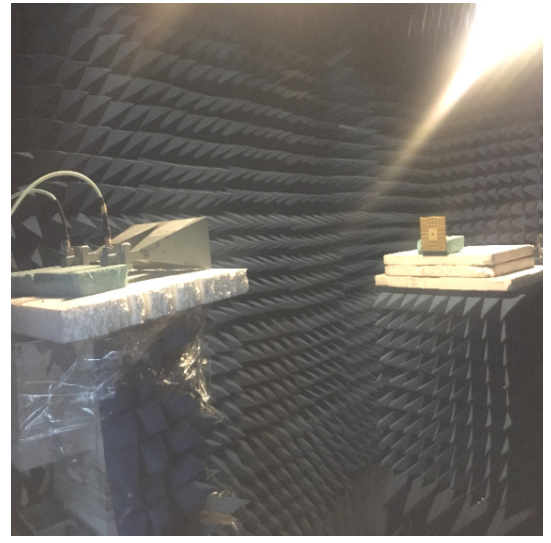

Fig. 16. Experimental setup for normal incidence.

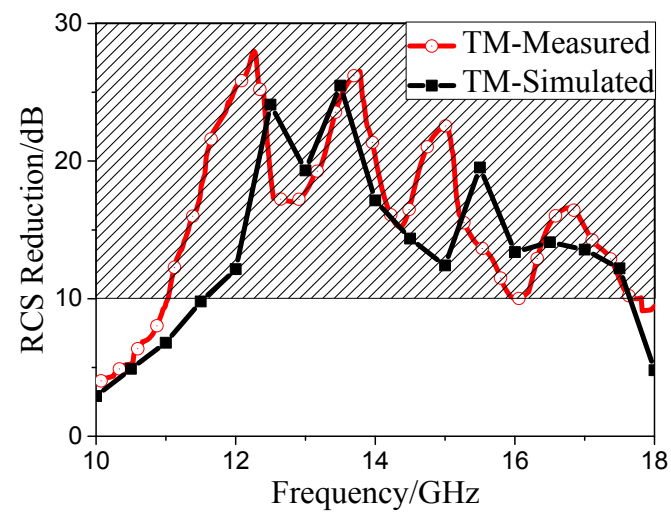

Fig. 17. RCS reduction under normal incidence with TM-polarization condition.

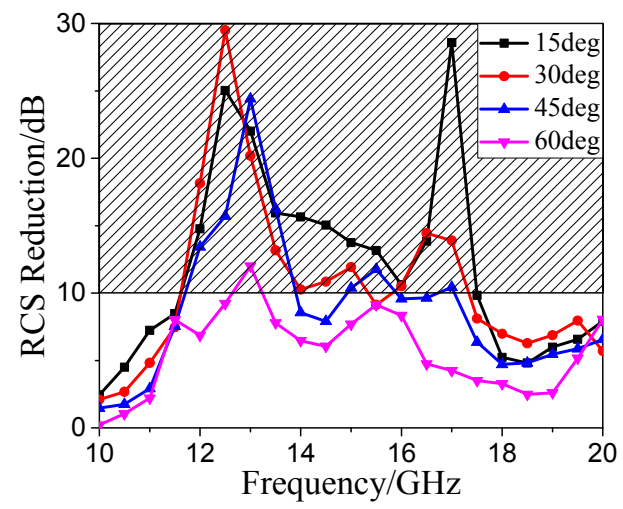

Fig. 18. RCS reduction at different incident angles with TM-polarization condition.

The whole measurement setup has been arranged in an anechoic chamber to avoid the interference of the surrounding objects, as shown in Fig. 16. Here, we only performed monostatic RCS measurements under normal incidence due to available test deficiencies. Two sets of linearly polarized horn antennas working with $8-12 \mathrm{GHz}$ and $12-18 \mathrm{GHz}$, utilized as the receiver and transmitter, and the sample antenna is placed in front of them. The RCS can be calculated by measuring the $S_{21}$ and all the results were derived from the Agilent N5245A vector network analyzer. Time Domain Gating of VNA is adopted to eliminate the jitter in $\mathrm{S}_{21}$ due to the near-field coupling between two horn antennas.
Finally, the measured and simulated RCS reduction result for a normal incident plane wave with TMpolarization is shown in Fig. 17.

It can be seen from Fig. 17 that the monostatic RCS is dramatically reduced in the frequency range of $11 \mathrm{GHz} 17.8 \mathrm{GHz}$, and an obvious in-band RCS reduction is also obtained with a maximum reduction of $28.5 \mathrm{~dB}$ at 12.2 GHz. The measured result is slightly different from that of simulation. This is probably caused by the dielectric loss and metal loss due to the fabrication, which affects the magnitude of AMC cells in the RCS reduction calculation. Besides, experimental errors and uncertainties during the measurement environment would also have influence on the measured results. To demonstrate the effectivities at oblique incidence angles, Figure 18 shows the simulated result under different angles.

According to the simulated results in Fig. 18, we can find that the $10 \mathrm{~dB}$ RCSR can be maintained well between $12 \mathrm{GHz}$ to $17 \mathrm{GHz}$ till the incidence angle come to $60 \mathrm{deg}$. Finally, the comparison between this paper and other relevant works is shown in Tab. 2.

\begin{tabular}{|c|c|c|c|c|}
\hline & $\begin{array}{c}\text { Resonant } \\
\text { Frequency } \\
(\mathrm{GHz})\end{array}$ & $\begin{array}{c}\text { Size of the } \\
\text { antenna }(\lambda)\end{array}$ & $\begin{array}{c}\text { Maximum gain } \\
\text { enhacenment } \\
(\mathrm{dB})\end{array}$ & $\begin{array}{c}\text { The fractional } \\
\text { bandwidth of } \\
\text { RCS reduction } \\
(\geq 10 \mathrm{~dB})\end{array}$ \\
\hline This paper & 12 & $3.6 \times 3.6 \times 0.096$ & 2 & $47.8 \%$ \\
\hline$[15]$ & 4.5 & $0.9 \times 0.9 \times 0.03$ & -0.1 & $29.6 \%$ \\
\hline$[16]$ & 9 & $3.2 \times 3.2 \times 0.16$ & None & $109 \%$ \\
\hline$[17]$ & 5.66 & $2.9 \times 2.9 \times 0.06$ & 5 & $12.3 \%$ \\
\hline
\end{tabular}

Tab. 2. Comparison of design performance with different AMC composite surfaces.

\section{Conclusion}

A broadband AMC structure with orthogonal slot embedding is presented in this paper. By adding slots on the arm of conventional dual-loop AMC unit, the in-phase reflection bandwidth of AMC is effectively expanded to $9 \mathrm{GHz} \sim 15 \mathrm{GHz}$ (50\% relative bandwidth). At the same time, by arranging two different AMC structures surrounding the patch antenna in a chessboard-like configuration, a simultaneous improvement of radiating and scattering performance are achieved over a wide frequency range. Both the simulation and experiment are utilized to validate the performances of the proposed antenna. The measured results are in a good agreement with simulated results. It is demonstrated that this antenna can be a good candidate for a stealth platform and the proposed broadband AMC structure can also be applied to other wideband antennas.

\section{References}

[1] WEN, D. E., HUANG, X. M., REN, P., et al. Study on the properties of the two-dimensional curved surface metamaterial. International Journal of Electronics and Communications, 2018 , vol. 83, p. 376-397. DOI: 10.1016/j.aeue.2017.10.009 
[2] ALU, A., ENGHETA, N. Guided modes in a waveguide filled with a pair of Single-Negative (SNG), Double-Negative (DNG), and/or Double-Positive (DPS) layers. IEEE Transaction on Microwave and Theory Techniques, 2004, vol. 52, no. 1, p. 199-210. DOI: 10.1109/TMTT.2003.821274

[3] Cheribi, H., GHANEM, F., KimOUCHE, H. Metamaterialbased frequency reconfigurable antenna. Electronics Letters, 2013, vol. 49, no. 5, p. 315-316. DOI: 10.1049/EL.2012.3651

[4] JOSE, J. Frequency selective bistable switching in metamaterial based photonic bandgapmedium. Optics Communications, 2014, vol. 328, p. 116-120. DOI: 10.1016/J.OPTCOM.2014.04.043

[5] HU, F. R., ZOU, T. B., QUAN, B. G., et al. Polarizationdependent terahertz metamaterial absorber with high absorption in two orthogonal directions. Optics Communications, 2014, vol. 332, p. 321-326. DOI: 10.1016/j.optcom.2014.06.017

[6] SIEVENPIPER, D., ZHANG, L. J., BROAS, R. F. J., et al. High impedance electromagnetic surfaces with a forbidden frequency band. IEEE Transactions on Microwave Theory and Techniques, 1999, vol. 47, no. 11, p. 2059-2074. DOI: 10.1109/22.798001

[7] RAJAGOPAL, S., R., CHENNAKESAVAN, G., C., SUBBURAJ, D. R. P., et al. A dual polarized antenna on a novel broadband multilayer Artificial Magnetic Conductor backed surface for LTE/CDMA/GSM base station applications. International Journal of Electronics and Communications, 2017, vol. 80, p. 73-79. DOI: 10.1016/j.aeue.2017.06.028

[8] WU, J. L., YANG, S. W., CHEN, Y. K., et al. A low profile dualpolarized wideband omnidirectional antenna based on AMC reflector. IEEE Transactions on Antennas and Propagation, 2017, vol. 65, no. 1, p. 368-374. DOI: 10.1109/TAP.2016.2631147

[9] LIN, J. D., QIAN, Z. P., CAO, W. Q., et al. A low-profile dualband dual-mode and dual-polarized antenna based on AMC. IEEE Antennas and Wireless Propagation Letters, 2017, vol. 16, p. 2473-2476. DOI: 10.1109/LAWP.2017.2724540

[10] ZHAI, H. Q., ZHANG, K. D., YANG, S., et al. A low-profile dual-band dual-polarized antenna with an AMC surface for WLAN applications. IEEE Antennas and Wireless Propagation Letters, 2017, vol. 16, p. 2692-2695. DOI: 10.1109/LAWP.2017.2741465

[11] YANG, W. C., CHEN, D. X., CHE, W. Q. High-efficiency highisolation dual-orthogonally polarized patch antennas using nonperiodic RAMC structure. IEEE Transactions on Antennas and Propagation, 2017; vol. 65, no. 2, p. 887-892. DOI: 10.1109/TAP.2016.2632700

[12] THUMMALURU, S. R., KUMAR, R., CHAUDHARY, R. K. Isolation enhancement and radar cross section reduction of MIMO antenna with frequency selective surface. IEEE Transactions on Antennas and Propagation, 2018, vol. 66, no. 3, p. 1595-1600. DOI: 10.1109/TAP.2018.2794417

[13] NARAYAN, S., SANGEETHA, B., SRUTHI, T. V., et al. Design of low observable antenna using active hybrid-element FSS structure for stealth applications. International Journal of Electronics and Communications, 2017, vol. 80, p. 137-143. DOI: 10.1016/j.aeue.2017.06.038
[14] LIU, Y., LI, K., JIA, Y. T., et al. Wideband RCS reduction of a slot array antenna using polarization conversion metasurfaces. IEEE Transactions on Antennas and Propagation, 2016, vol. 64, no. 1, p. 326-331. DOI: 10.1109/TAP.2015.2497352

[15] ZOU, S. X., WEI, J. L., MAN, X. Wideband RCS reduction of patch antenna using PRRS. Electronic Letters, 2017, vol. 53, no. 8, p. 522-524. DOI: 10.1049/el.2016.2640

[16] XUE, J. J., JIANG, W., GONG, S. X. Wideband RCS reduction of slot-coupled patch antenna by AMC structure. Electronic Letters, 2017, vol. 53, no. 22, p. 1454-1456. DOI: 10.1049/el.2017.2587

[17] LIU, X., GAO, J., CAO, X. Y., et al. A high-gain and lowscattering waveguide slot antenna of artificial magnetic conductor octagonal ring arrangement. Radioengineering, 2016, vol. 25, no. 1, p. 46-52. DOI: 10.13164/re.2016.0046

[18] ZHENG, Y. J., GAO, J., ZHOU, Y. L., et al. Metamaterial-based patch antenna with wideband RCS reduction and gain enhancement using improved loading method. IET Microwaves Antennas and Propagations, 2017, vol. 11, no. 9, p. 1183-1189. DOI: 10.1049/iet-map.2016.0746

[19] ZHAO, Y., CAO, X. Y., GAO, J., et al. Broadband radar absorbing material based on orthogonal arrangement of CSRR etched artificial magnetic conductor. Microwave and Optical Technology Letters, 2014, vol. 56, no. 1, p. 158-161. DOI: 10.1002/mop.28033

[20] PAQUAY, M., IRIARTE, J. C., EDERRA, I., et al. Thin AMC structure for radar cross-section reduction. IEEE Transactions on Antennas and Propagation, 2007, vol. 55, no. 12, p. 3630-3638. DOI: 10.1109/TAP.2007.910306

\section{About the Authors ...}

Yue GE was born in 1994. He received his B.S. degree in Electronic Science and Technology from the Nanjing University of Aeronautics and Astronautics (NUAA), Nanjing, China in 2016. Currently he is working towards the M.S. degree in the NUAA. His research interests include metamaterial and modern antenna design.

Yongjiu ZHAO was born in 1964. He received his Ph.D. degree in Xidian University, Xi'an, China. He is currently a professor with the College of Electronic Information Engineering, Nanjing University of Aeronautics and Astronautics, Nanjing, Jiangsu, China. His research interests include microwave circuits design, wireless communication and wave propagation.

Jiaqing CHEN was born in 1989. He is working towards his Ph.D. degree in the Nanjing University of Aeronautics and Astronautics, Nanjing, China. Currently his research interests include metasurface and metamaterial design. 